ISSN 0103-8478

\title{
Aspectos nutricionais e ambientais da obesidade canina
}

\author{
Nutritional and environment aspects of canine obesity
}

\author{
Karina Preising Aptekmann ${ }^{\mathrm{I}}$ Weslem Garcia Suhett ${ }^{\mathrm{II}}$ Aguinaldo Francisco Mendes Junior ${ }^{\mathrm{II}}$ \\ Graziela Baroni Souza ${ }^{I I}$ Ana Paula Pinto Araújo Tristão ${ }^{I I I}$ Felipe Kunz Adamsv \\ Caroline Godoi Aoki ${ }^{\mathrm{IV}}$ Reinaldo Juan Garrido Palacios Junior ${ }^{\mathrm{IV}}$ Aulus Cavalieri Carciofi ${ }^{\mathrm{V}}$ \\ Mirela Tinucci-Costa ${ }^{\mathrm{v}}$
}

\section{RESUMO}

A obesidade é a doença nutricional mais frequente em cães, leva a uma série de alterações nas funções corporais $e$ limita a longevidade dos animais. Vários fatores contribuem para o desenvolvimento da obesidade em cães, como genética, raça, idade, falta de atividade física, composição calórica dos alimentos, tipo e a forma de alimentação, distúrbios hormonais, medicamentos e fatores relacionados com os proprietários. Devido à falta de estudos sobre os aspectos nutricionais e ambientais envolvidos no desenvolvimento da obesidade canina, o presente trabalho objetivou obter estas informações por meio de enquetes a proprietários. Foram realizadas 254 entrevistas com proprietários de cães com sobrepeso ou obesos, atendidos em Hospitais Veterinários Universitários. Selecionaram-se cães com escore de condição corporal (ECC) de 6 a 9, de acordo com escala descrita por LAFLAMME (1997). A maioria dos cães acometidos eram fêmeas adultas castradas, sem raça definida (33\%) ou Poodles (17\%). De acordo com $36 \%$ dos respondentes, o excesso de alimento era a possível razão do ganho de peso dos animais. Apenas 52\% dos proprietários tentaram realizar controle de peso do seu animal, principalmente reduzindo a quantidade de alimento. Não houve correlação entre a condição corporal do proprietário, nível de escolaridade, renda familiar e ambiente domiciliar dos proprietários com o ECC dos animais. As informações obtidas podem auxiliar na identificação dos fatores nutricionais e ambientais envolvidos no desenvolvimento da obesidade em cães, podendo adotar medidas preventivas e desenvolver melhores estratégias para o tratamento.

Palavras-chave: cão, enquete, alimentação, epidemiologia.

\section{ABSTRACT}

Obesity is the most common nutritional disease in dogs and may cause many changes in body systems and limit the longevity of the animals. Many factors contribute to the development of obesity in dogs, as genetics, breed, age, lack of physical activity, caloric food composition, type and form of nutrition, hormonal disorders, medicine, and factors related to the owners. Due to the lack of studies on the nutritional and environmental aspects involved in the development of canine obesity, the present study aimed to obtain information through owners surveys. Interviews with 254 owners of overweight or obese dogs were conducted at University Veterinary Hospitals. Dogs were selected through body condition score (BCS) from 6 to 9, according to the scale described by LAFLAMME (1997). The data were coded, tabulated and submitted to frequency analysis and correlation (Spearman Test). Most affected dogs were castrated adult female of mixed breeds (33\%) or poodles (17\%). For most respondents (36\%), food excess was the possible reason for the weight gain of pets. Only half of the owners have tried to achieve weight control of their pet, especially reducing the amount of food. There was no correlation between body condition of the owner, educational level, family income and home environment of the owners with pets BCS. Information obtained may help identifying nutritional and environmental factors involved in the development of obesity in dogs and can provide better preventive measures and strategies for treatment.

Key words: dog, survey, feeding, epidemiology.

\section{INTRODUÇÃO}

A obesidade é um distúrbio nutricional que muitas vezes não é diagnosticado como uma doença. Em cães, ocorre quando o peso está pelo menos $15 \%$ acima do ideal, consequente do acúmulo excessivo de

IDepartamento de Medicina Veterinária, Centro de Ciências Agrárias, Universidade Federal do Espírito Santo (UFES), Alto Universitário, s/no, CP 16, Guararema, 29500-000, Alegre, ES, Brasil. E-mail: kapreising@ yahoo.com.br. Autor para correspondência.

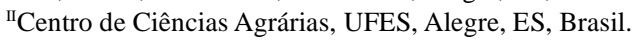

"IIPrograma de Residência em Medicina Veterinária, Faculdade de Ciências Agrárias e Veterinárias (FCAV), Universidade Estadual Paulista "Júlio de Mesquita Filho" (UNESP), Jaboticabal, SP, Brasil.

Iv Programa de Pós-graduação em Medicina Veterinária, FCAV, UNESP, Jaboticabal, SP, Brasil.

v Departamento de Clínica e Cirurgia Veterinária, UNESP, Jaboticabal, SP, Brasil.

$$
\begin{array}{cc}
\text { Recebido 16.04.13 Aprovado 18.04.14 Devolvido pelo autor 01.08.14 } \\
\text { CR-2013-0524 }
\end{array}
$$


gordura corporal (BURKHOLDER \& TOLL, 2000). A obesidade pode aumentar o risco de aparecimento de várias complicações (DIEZ \& NGUYEN, 2006), tornando-se prejudicial à saúde do cão, e pode resultar em redução da sua qualidade de vidae da longevidade (MARKWELL et al., 1990; LAWLER et al., 2005). É uma enfermidade universal de prevalência crescente, sendo um dos principais problemas de saúde pública nos tempos atuais (MANCINI, 2001). A manutenção do peso corporal ideal é fator determinante para manutenção da saúde, bem estar e qualidade de vida. Em situações que ocorrem desequilíbrio entre a ingestão calórica e a diminuição da prática da atividade física ocorre a obesidade (SALVE, 2006).

Assim como nos humanos, o excesso de peso corporal vem se tornando uma preocupação frequente na medicina veterinária (BLAND et al., 2010). Fatores relacionados com os proprietários também são identificados como fatores de risco no desenvolvimento da obesidade em cães (LUND et al., 2006; BLAND et al., 2010; COURCIER, et al., 2010). O hábito alimentar dos cães e gatos passou por mudanças nas últimas décadas, devido à influência do homem; tornou-se comum a alimentação desses animais com petiscos e guloseimas.

Desta forma, tendo em vista a importância do reconhecimento da obesidade em cães, as consequências causadas e a falta de estudos no país, este estudo teve como objetivo realizar uma enquete, por meio de entrevistas aos proprietários, a fim de obter informações sobre o perfil dos animais obesos e os aspectos nutricionais e ambientais envolvidos no desenvolvimento da obesidade em cães.

\section{MATERIAL E MÉTODOS}

Para realização da enquete, foram realizadas 254 entrevistas com proprietários de cães com sobrepeso ou obesos, atendidos nos Hospitais Veterinários da Universidade Estadual Paulista, em Jaboticabal - SP e Universidade Federal de Viçosa - MG. Os proprietários foram esclarecidos sobre a pesquisa e voluntariamente responderam ao questionário.

Para a seleção dos cães, foi utilizada a escala de classificação de escore de condição corporal (ECC) de nove pontos, descrita por LAFLAMME (1997), sendo selecionados os cães com ECC entre 6 e 9. Os proprietários também foram questionados quanto à condição corporal de seus cães. O questionário foi composto por 22 perguntas objetivas de múltipla escolha e de fácil compreensão. As perguntas foram lidas pelo entrevistador e, quando necessário, foram especificadas as alternativas para a resposta.

Posteriormente, os dados foram codificados e tabulados em planilhas do Microsoft Excel e foi feita a análise e descrição dos resultados. Foi realizada uma análise estatística de frequência. Para as correlações entre as questões, foi utilizado o coeficiente de correlação de Spearman. Utilizou-se nível de significância de 5\%.

\section{RESULTADOS E DISCUSSÃO}

A distribuição do ECC, determinado pelo entrevistador, dos 254 cães com sobrepeso ou obesos incluídos na enquete foi a seguinte: ECC 6 (19\%); ECC 7 (29\%); ECC 8 (17\%) e ECC 9 (35\%). Na opinião dos proprietários, os cães foram considerados magros (1\%), com condição corporal ideal (31\%), com sobrepeso (41\%) ou obesos (27\%). Foi encontrada uma correlação positiva entre a opinião dos proprietários a respeito da condição corporal do animal e o ECC encontrado $(\mathrm{P}<0,0001 ; \mathrm{R} 2=0,55)$. Entretanto, evidenciou-se uma discrepância na avaliação dos cães obesos pelo entrevistador (52\% com ECC 8 ou 9) e pelos proprietários (27\%), demonstrando que a obesidade pode ser subestimada pela percepção dos proprietários (Figura 1). Foram realizadas perguntas sobre a opinião dos proprietários a respeito da sua própria condição corporal. Dos proprietários, $45 \%$ se consideraram com peso ideal, $36 \%$ com sobrepeso, $10 \%$ obeso e $9 \%$ magro (Figura 1). Não houve correlação entre a condição corporal do proprietário e o ECC dos animais $(\mathrm{P}=0,55 ; \mathrm{R} 2=0,038)$.

$\mathrm{Na}$ população estudada, os cães apresentavam, em média, 7 anos de idade. Esses resultados permitiram determinar que a maioria dos cães era adulto, assim como evidenciado em outros estudos epidemiológicos (LAZZAROTO, 1999; DIEZ \& NGUYEN, 2006; COURCIER et al., 2010). Sabe-se que, à medida que o animal envelhece, ocorre diminuição dos gastos de energia e do metabolismo basal. Além disso, há uma inversão da massa magra. que é substituída por massa gorda. Sendo assim, aconselha-se reduzir entre 10 a $15 \%$ o consumo de energia a partir dos sete anos, de acordo com a condição corporal do animal (DIEZ \& NGUYEN, 2006).

Quanto ao sexo de cães, foi encontrado: fêmea castrada (34\%), fêmea não castrada (28\%), macho não castrado (28\%) e macho castrado (10\%), mostrando uma maior prevalência de fêmeas castradas. Estes dados corroboram os estudos de JERICÓ \& SCHEFER (2002), podendo esse fato 


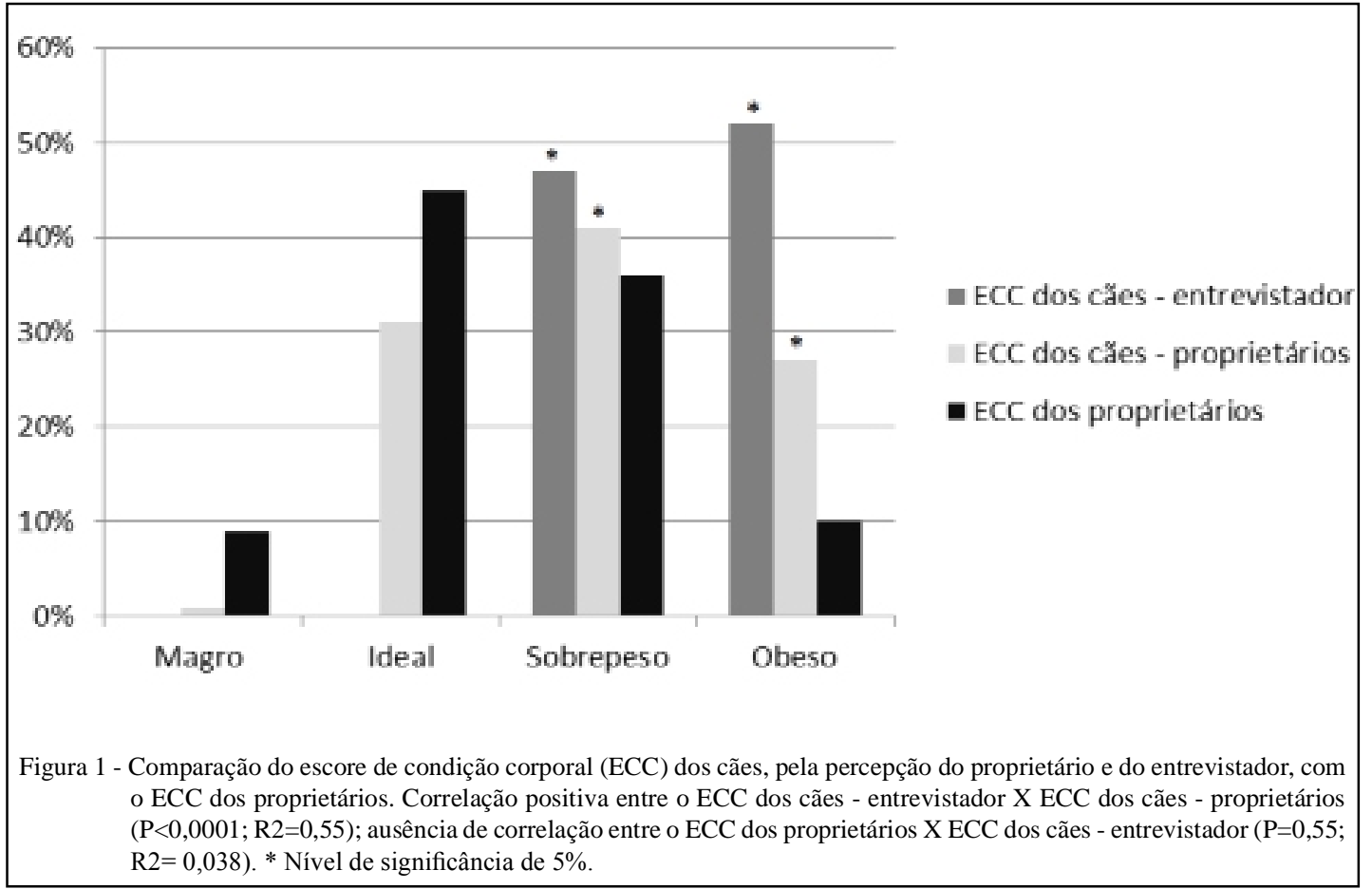

ser explicado, pois as fêmeas apresentam uma menor taxa metabólica basal do que os machos e a gonadectomia causa uma desacereleração metabólica que predispõe ao ganho de peso (BURKHOLDER \& TOLL, 2000; KIL \& SWANSON, 2010). Observou-se que as fêmeas castradas podem ter mais predisposição à obesidade ou sobrepeso do que os machos castrados, fato também evidenciado por COURCIER et al. (2010).

Na população estudada, as raças de cães obesos ou com sobrepeso foram as seguintes: Poodle (15\%), Cocker Spaniel Inglês (8\%), American Pit Bull Terrier (8\%), Labrador Retriever (7\%), Teckel $(5 \%)$, Rottweiler (4\%), Boxer (4\%), Schnauzer (3\%), Beagle (3\%) e demais raças (15\%). Além disso, $28 \%$ dos animais não apresentavam raça definida. As raças de cães Cocker Spaniel Inglês e Labrador Retriever já foram descritas como mais predispostas à obesidade no país (JERICÓ \& SCHEFFER, 2002; COLLIARD et al., 2006; COURCIER et al., 2010).

Com relação ao apetite, $49 \%$ dos proprietários consideravam que o cão apresentava um apetite normal, $48 \%$ aumentado e $4 \%$ diminuído. Apesar da fraca correlação, observou-se que a presença de cães com ECC 8 ou 9 se correlacionou com os animais que apresentavam maior apetite $(\mathrm{P}=0,001$; $\mathrm{R} 2=-0,20)$. Os entrevistados relataram que a possível razão do excesso de peso foi: excesso de alimento (28\%), pouca atividade física (13\%), castração
(10\%), doença (7\%), genética $(7 \%)$ e medicamento (4\%). Contudo, não há embasamento científico para afirmar que a maior causa de obesidade é de origem alimentar. A maioria dos proprietários (69\%) afirmou ter outros cães ou gatos em casa, porém, não houve correlação entre a presença de outros animais no ambiente com o ECC dos obesos ( $\mathrm{P}=1,0 ; \mathrm{R} 2=-0,12)$.

Quase todos os proprietários $(98 \%)$ afirmaram saber que a obesidade é prejudicial à saúde do animal, porém somente metade deles $(51 \%)$ já pediu orientação ao veterinário para controlar o peso do animal. Observou-se que há uma consciência por parte dos proprietários quanto aos riscos da obesidade, entretanto, poucos procuram orientação para tratar o problema. Este fato também foi observado em outro estudo, no qual não houve correlação significativa da consciência do proprietário sobre os riscos da obesidade em cães com a presença de obesidade ou sobrepeso (COURCIER et al., 2010).

Metade dos proprietários já havia tentado reduzir o peso do seu animal. Para isto, os proprietários adotaram várias medidas, dentre elas: redução na quantidade de alimento (66\%), mudança na dieta $(53 \%)$, retirada de petiscos $(35 \%)$, exercício físico $(28 \%)$ e medicamentos $(0,8 \%)$. Dentre os proprietários que não tentaram reduzir o peso de seus animais, a maioria (84\%) estava disposta a iniciar o tratamento para o controle de peso dos cães. O manejo dietético é o fator principal para o tratamento 
da obesidade em cães, possuindo como métodos complementares a instituição de exercício físico e abordagem ao proprietário (GERMAN, 2006). Apesar dos resultados apontarem que a principal forma de tratamento estabelecida pelos proprietários foi a redução na quantidade do alimento, sabese que uma dieta apropriada para perda de peso deve ser estabelecida, uma vez que é totalmente contra indicado alcançar uma restrição energética simplesmente reduzindo a quantidade da ração de manutenção que é normalmente consumida pelo animal, pois pode levar a deficiências nutricionais importantes sem alcançar o sucesso na terapia (DIEZ \& NGUYEN, 2006, LAFLAMME, 2006).

Atualmente, a facilidade de acesso à informação auxilia os proprietários na escolha do tipo de alimento. Os meios mais utilizados para obtenção de informações pelos proprietários foram: consulta a veterinários/ zootecnistas (69\%), lojas agropecuárias ou pet shops (18\%); internet (11\%); revistas/ livros (5\%); estudantes de veterinária/zootecnia (4\%); televisão/rádio (3\%); e outros $(11 \%)$.

A alimentação é um dos fatores que está diretamente relacionado com a condição corporal do animal. Os tipos de alimentos fornecidos aos cães deste estudo estão dispostos na tabela 1. Vinte e cinco porcento dos proprietários forneciam mais de um tipo de alimento a seus cães. A ração seca de manutenção foi a mais utilizada pelos proprietários, sendo fornecida a $94 \%$ dos cães. As rações light e terapêuticas têm sido recomendadas para cães para o tratamento da obesidade (BURKHOLDER \& TROLL, 2000). Entretanto, nossos resultados mostraram que somente $25 \%$ dos proprietários forneciam rações secas hipocalóricas aos animais obesos. Quatro por cento dos proprietários de cães afirmaram fornecer ração úmida. Trinta e cinco por cento dos proprietários afirmaram fornecer comida caseira aos seus animais, sendo que metade deles preparava o alimento especificamente para o cão e outra metade oferecia o alimento preparado para o consumo familiar. A ração comercial foi considerada como o tipo de alimento mais saudável para o consumo de seus cães pela maioria dos proprietários (89\%), quando comparada com a comida caseira.

Foi constatado que os proprietários têm o hábito de oferecer aos seus animais outros tipos de alimento além da ração ou comida caseira. Observouse que $49 \%$ dos cães recebiam petiscos diariamente, $28 \%$ nunca recebiam, $18 \%$ algumas vezes ao mês e $8 \%$ algumas vezes na semana. Sabe-se que o oferecimento de petiscos é considerado um fator de risco para o desenvolvimento da obesidade (COLLIARD, 2006; COURCIER et al., 2010). Apesar disso, observou-se que muitos cães (28\%) apresentavam excesso de peso mesmo sem se alimentar de petiscos, provavelmente relacionando-se a outras causas de obesidade.

Houve grande variação na frequência com que os alimentos eram oferecidos aos animais: uma vez ao dia ( $8 \%$ ), duas vezes ao dia (58\%), três vezes ao dia $(14 \%)$, mais de três vezes ao dia $(3 \%)$ e alimento à vontade $(18 \%)$. De acordo com os resultados, observou-se que os cães obesos ou com sobrepeso eram alimentados preferencialmente duas vezes ao dia. Contudo, uma porcentagem considerável dos proprietários ainda fornecia alimento à vontade, $\mathrm{o}$ que também pode ser considerado um fator de risco para o desenvolvimento da obesidade nesses animais (DIEZ \& NGUYEN, 2006). Não houve correlação entre a frequência de alimentação e o $\mathrm{ECC}(\mathrm{P}=0,36$; $\mathrm{R} 2=0,05)$, como também observado em outro estudo (COURCIER et al., 2010).

Segundo informações obtidas pelos proprietários que forneciam ração, a quantidade de alimento oferecido era definida por meio de: recomendações do veterinário/zootecnista (39\%), até o animal parar de se alimentar $(26 \%)$, instruções do pacote $(22 \%)$, pela condição corporal do animal $(9 \%)$

Tabela 1 - Dieta de manutenção básica dos cães obesos ou com sobrepeso (n=254).

\begin{tabular}{|c|c|c|c|}
\hline Dieta & Total & Tipo & Porcentagem \\
\hline \multirow{3}{*}{ Ração seca } & \multirow{3}{*}{$94 \%$} & Manutenção & $76 \%$ \\
\hline & & Light & $13 \%$ \\
\hline & & Terapêutica & $12 \%$ \\
\hline \multirow{3}{*}{ Ração úmida } & \multirow{3}{*}{$4 \%$} & Manutenção & $82 \%$ \\
\hline & & Light & $9 \%$ \\
\hline & & Terapêutica & $9 \%$ \\
\hline \multirow{2}{*}{ Comida caseira } & \multirow{2}{*}{$35 \%$} & Preparada para o animal & $50 \%$ \\
\hline & & Preparada para consumo humano & $50 \%$ \\
\hline
\end{tabular}


e outros (15\%). Esses resultados mostraram que, apesar de 39\% dos proprietários terem sido instruídos por profissionais, muitos ainda estabelecem a quantidade de alimento a ser oferecida a seus cães de forma subjetiva.

De acordo com os proprietários que forneciam ração aos animais, a escolha desse tipo de alimento foi determinada por considerarem mais saudável $(64 \%)$, por recomendação do veterinário e/ou zootecnista (37\%), por facilidade (27\%), dentre outras razões. No momento de adquirir a ração, os proprietários levavam em consideração, principalmente, a aceitação pelo animal (47\%), a marca $(35 \%)$, recomendação do veterinário e/ou zootecnista (35\%), o custo (22\%) e a recomendação para a raça $(17 \%)$. Na opinião dos proprietários, a escolha pelo fornecimento de comida caseira aos animais se devia à preferência do animal (61\%), facilidade $(9 \%)$ ou por considerarem mais saudável que a ração $(9 \%)$. Verificou-se que a escolha por ração ou comida caseira se deveu especialmente pela aceitação do animal.

Foram realizadas perguntas sobre a escolaridade, renda familiar e ambiente domiciliar na tentativa de correlacionar com as demais informações obtidas no questionário. A maioria dos entrevistados (60\%) possuía ensino superior completo ou incompleto, $27 \%$ possuíam ensino médio completo ou incompleto, $5 \%$ cursaram entre a quinta e oitava série do ensino fundamental e $8 \%$ eram analfabetos ou cursaram até a quarta série do ensino fundamental. Quanto à renda média da família (em salários mínimos), 59\% recebia acima de 4 salários, $18 \%$ de 2 a 4 salários, $12 \%$ de 1 a 2 salários, $1 \%$ menos que 1 salário. Dez por cento se negou a responder ou não soube informar. Dentre os entrevistados, $92 \%$ viviam na área urbana e 8\% na área rural.

Estudo realizado por COURCIER et al. (2010) concluiu que o excesso de peso do animal foi significativamente associado com a renda e a idade do proprietário, no qual quanto menor a renda e maior a idade do proprietário, maior a probabilidade do animal desenvolver a obesidade. Entretanto, no presente estudo, não foi observada correlação entre a idade do proprietário $(\mathrm{P}=0,59 ; \quad \mathrm{R} 2=0,03)$, sexo do proprietário $(\mathrm{P}=0,36$; $\mathrm{R} 2=0,05)$, nível de escolaridade $\mathrm{P}=0,68 ; \mathrm{R} 2=-0,02)$, renda familiar $(\mathrm{P}=0,96 ; \mathrm{R} 2=0,003)$ e ambiente domiciliar $(\mathrm{P}=0,86 ; \mathrm{R} 2=-0,01)$ e o $\mathrm{ECC}$ dos animais.

\section{CONCLUSÃO}

As informações obtidas nesta enquete podem auxiliar médicos veterinários na identificação dos fatores nutricionais e ambientais envolvidos no desenvolvimento da obesidade em cães, podendo adotar medidas preventivas e desenvolver melhores estratégias para o tratamento da obesidade. $\mathrm{O}$ fato de que muitos proprietários não identificam o sobrepeso em seus animais, ou não buscam tratamento, torna importante o reconhecimento deste distúrbio nutricional por veterinários e estabelecimento de dieta adequada para redução de peso.

\section{COMITÊ DE ÉTICA E BIOSSEGURANÇA}

O estudo foi aprovado pela Comissão de Ética no Uso de Animais (CEUA) da Universidade Federal do Espírito Santo (UFES), sob protocolo n.086/2011.

\section{REFERÊNCIAS}

BLAND, I.M. et al. Dog obesity: veterinary practices and owners opinions on cause and management. Preventive Veterinary Medicine, v.94, p.310-315, 2010. Disponível em: <http://dx.doi. org/10.1016\%2Fj.prevetmed.2010.01.013>. Acesso em: 20 jul. 2012. doi: 10.1016/j.prevetmed.2010.01.013.

BURKHOLDER, W.J.; TOLL, P.W. Obesity. In: HAND, M.S. et al. Small animal of clinical nutrition. 4.ed. Kansas: Mark Morres Institute, 2000. p.401-430.

COLLIARD, L. et al. Risk factors for obesity in dogs in France. Journal of Nutrition, v.136, p.1951S-1954S, 2006. Disponível em: <http://jn.nutrition.org/content/136/7/1951S.full>. Acesso em: 20 jul. 2012.

COURCIER, E.A. et al. An epidemiological study of environmental factors associated with canine obesity. Journal of Small Animal Practice, v.51, p.362-367, 2010. Disponível em: <http://dx.doi. org/10.1111\%2Fj.1748-5827.2010.00933>. Acesso em: 20 jul. 2012. doi: 10.1111/j.1748-5827.2010.00933.

DIEZ, M.; NGUYEN, P. Obesity: epidemiology, pathophysiology and management of the obese dog. In: PIBOT, P. et al. Encyclopedia of canine clinical nutrition. France: Aniwa SAS, 2006. p.2-57.

GERMAN, A.J. The growing problem of obesity in dogs and cats. Journal of Nutrition, v.136, p.1940S-1946S, 2006. Disponível em: <http://jn.nutrition.org/content/136/7/1940S.abstract>. Acesso em: 20 jul. 2012.

JERICÓ, M.M.; SCHEFFER, K.C. Aspectos epidemiológicos dos cães obesos na cidade de São Paulo. Revista Clínica Veterinária, v.37, p.25-29, 2002. Disponível em: <http://www. revistaclinicaveterinaria.com.br/edicao/2002/marco-abril.html>. Acesso em: 27/05/2014

KIL, D.Y.; SWANSON, K.S. Endocrinology of obesity. Veterinary Clinics of North America - Small Animal Practice, v.40, p.205219, 2010. Disponível em: <http://dx.doi.org/10.1016\% $2 \mathrm{Fj}$. cvsm.2009.10.004>. Acesso em: 20 jul. 2012. doi: 10.1016/j. cvsm.2009.10.004

LAFLAMME, D. Development and validation of a body condition score system for dogs. Canine Practice, v.22, p.10-15, 
1997. Disponível em: <http://agris.fao.org/agris-search/search. do?recordID=US9742264>.

LAFLAMME, D.P. Understanding and managing obesity in dogs and cats. Veterinary Clinics of North America - Small Animal Practice, v.36, p.1283-1295, 2006. Disponível em: <http://www. ncbi.nlm.nih.gov/pubmed/17085235>. Acesso em: 20 jul. 2012. doi: 10.1016/j.cvsm.2006.08.005.

LAWLER, D.F. et al. Influence of lifetime food restriction on causes, time, and predictors of death in dogs. Journal of the American Veterinary MedicineAssociaton, v.226, p.225-231, 2005. Disponível em: 〈http://dx.doi.org/10.2460\% 2Fjavma.2005.226.225>. Acesso em: 23 jul. 2012. doi:10.2460/Fjavma.2005.226.225.

LAZZAROTO, J.J. Relação entre aspectos nutricionais e obesidade em pequenos animais. Revista Universidade Alfenas, v.5, p.33-35, 1999. Disponível em: <http://www. unifenas.br/pesquisa/download/ArtigosRev1_99/pag33-35. pdf>. Acesso em: 23 jul. 2012.
LUND, E.M. et al. Prevalence and risk factors for obesity in adult dogs from private US veterinary practices. Journal of Applied Research in Veterinary Medicine veterinary, v.4, p.177-186, 2006. Disponível em: <http://www.jarvm.com/articles/Vol4Iss2/ Lund.pdf $>$. Acesso em 23 de jul de 2012.

MANCINI, M.C. Obstáculos diagnósticos e desafios terapêuticos no paciente obeso. Arquivos Brasileiros de Endocrinologia \& Metabologia, v.45, p.584-608, 2001. Disponível em: <http://www. scielo.br/scielo.php?pid=S0004-27302001000600013\&script $=$ sci_ arttext>. Acesso em: 23 jul. 2012.

MARKWELL, P.J. et al. Obesity in the dog. Journal of Small Animal Practice, v.31, p.533-537, 1990. Disponível em: <http:// onlinelibrary.wiley.com/doi/10.1111/j.1748-5827.1990.tb00680.x/ abstract>. Acesso em: 23 jul. 2012.

SALVE, M.G.C. Obesidade e peso corporal: riscos e consequências. Movimento \& Percepção, v.6, n.8, p.29-48, 2006. Disponível em: <http://portalsaudebrasil.com/artigospsb/obes204.pdf >. Acesso em: 23 jul. 2012. 